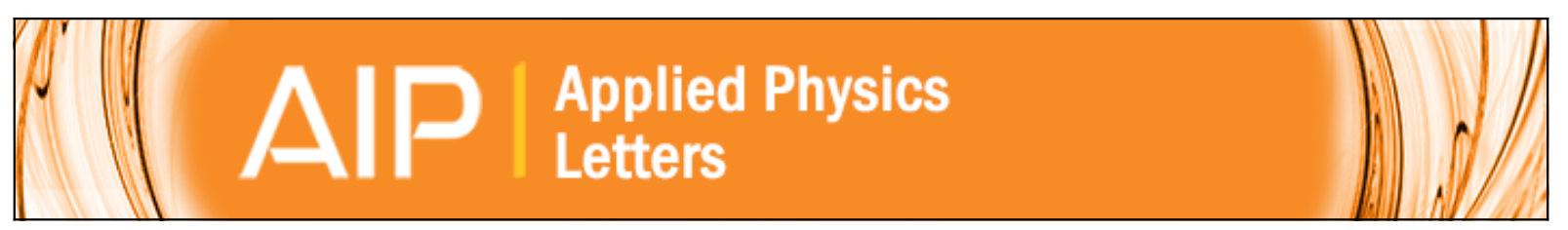

\title{
Self-biased converse magnetoelectric effect
}

Su Chul Yang, Kyung-Hoon Cho, Chee-Sung Park, and Shashank Priya

Citation: Applied Physics Letters 99, 202904 (2011); doi: 10.1063/1.3662420

View online: http://dx.doi.org/10.1063/1.3662420

View Table of Contents: http://scitation.aip.org/content/aip/journal/apl/99/20?ver=pdfcov

Published by the AIP Publishing

\section{AlP Re-register for Table of Content Alerts}




\title{
Self-biased converse magnetoelectric effect
}

\author{
Su Chul Yang, Kyung-Hoon Cho, ${ }^{\text {a) }}$ Chee-Sung Park, and Shashank Priya ${ }^{\text {a) }}$ \\ Center for Energy Harvesting Materials and Systems (CEHMS), Virginia Tech, Blacksburg, \\ Virginia 24061, USA
}

(Received 25 August 2011; accepted 29 October 2011; published online 17 November 2011)

\begin{abstract}
In this letter, we investigate the direct magnetoelectric (DME) and converse magnetoelectric (CME) effects in three-phase metal-ceramic laminate composites. Longitudinally poled and transversely magnetized (L-T) laminate was fabricated by bonding nickel plates between the two particulate magnetoelectric (ME) composite layers of composition $0.8\left(0.948 \mathrm{~K}_{0.5} \mathrm{Na}_{0.5} \mathrm{NbO}_{3}-0.052 \mathrm{LiSbO}_{3}\right)$ - $0.2\left(\mathrm{Ni}_{0.8} \mathrm{Zn}_{0.2} \mathrm{Fe}_{2} \mathrm{O}_{4}\right)$ (KNNLS-NZF). Under off-resonance condition, the laminates exhibited hysteretic DME and CME responses as a function of applied bias field $\left(H_{\text {bias }}\right)$. Self-biased effect characterized by non-zero ME response at zero $H_{\text {bias }}$ was observed. The self-biased DME and CME properties were found to be enhanced under resonance conditions. Without external $H_{\text {bias }}$, magnetic induction switching was possible by applying $\mathrm{AC}$ voltage. These results provide the possibility of using self-biased CME effect in electrically controlled memory devices and magnetic flux control devices. (C) 2011 American Institute of Physics. [doi:10.1063/1.3662420]
\end{abstract}

Magnetoelectric (ME) multiferroics exhibit simultaneous ferroelectric and ferromagnetic properties and have been of interest for applications such as magnetic field sensors, communication components, and electric-write/magneticread memory devices. ${ }^{1-4}$ Prior research on ME materials has shown that the composite structures consisting of ferromagnetic and ferroelectric phases possess much stronger ME coupling effect than single phase multiferroics. ${ }^{5}$ The ME effect can be classified as direct magnetoelectric (DME) and converse magnetoelectric (CME). DME effect is defined as change in electric polarization in response to applied magnetic field while CME effect is defined as change in magnetization in response to applied electric field. ${ }^{5,6}$ Most of the previous research on ME composites have focused on the DME effect. Field conversion ratio between applied ac magnetic field $\left(H_{\mathrm{ac}}\right)$ and induced ac electric field $\left(E_{\mathrm{ac}}\right)$, $\alpha_{\mathrm{DME}}=\delta E_{\mathrm{ac}} / \delta H_{\mathrm{ac}}$, has been frequently used as a figure-ofmerit for the DME effect. Many studies have revealed that bias magnetic field $\left(H_{\text {bias }}\right)$ is essential along with $H_{\mathrm{ac}}$ in order to enhance the DME effect and maximum in $\alpha_{\mathrm{DME}}$ is obtained at optimum $H_{\text {bias }}$. The $H_{\text {bias }}$ dependence of $\alpha_{\text {DME }}$ is related to the magnetostriction $(\lambda)$ of ferromagnetic phase and the behavior of $\alpha_{\mathrm{DME}}$ with respect to bias field has been experimentally confirmed to be very similar to that of piezomagnetic coefficient ( $\left.q=d \lambda / d H_{\text {bias }}\right)$ of ferromagnetic phase. Depending upon the composition and dimension of the ferromagnetic material, the maximum $\alpha_{\mathrm{DME}}$ is obtained at different $H_{\text {bias }}$ ranging from few Oe to few kOe., ${ }^{3,5,7}$ Since the requirement of additional large $H_{\text {bias }}$ can be problematic for device fabrication, efforts to reduce required $H_{\text {bias }}$ have been emphasized. ${ }^{7}$ Most ferromagnetic materials exhibit a very small slope in their magnetostriction curve near zero bias field $\left(H_{\text {bias }}=0\right)$ resulting in almost zero $q$ value and consequently negligible $\alpha_{\text {DME }}$. However, recent studies have revealed that by employing heterogeneous ferromagnetic structure, a large $\alpha_{\mathrm{DME}}$ value can be obtained in the absence

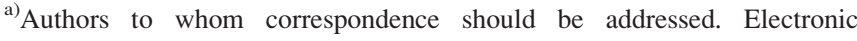
addresses: gomoo97@gmail.com and spriya@vt.edu.
}

of $H_{\text {bias}}$, so called self-biased DME effect. ${ }^{8-10}$ The selfbiased DME effect was observed when the laminates were operated in bending mode and consisted of dissimilar or graded magnetic materials that resulted in built-in magnetic bias. The discovery of self-biased DME effect is encouraging for applications such as self-biased magnetic field sensor.

Very few studies have investigated the CME effect in ME composites, in particular, self-biased CME effect has never been reported to date even though it has significant importance for electrically controllable magnetic devices. ${ }^{11,12}$ In this letter, we report that the CME effect $\left(\alpha_{\mathrm{CME}}=\delta B_{\mathrm{ac}}\right)$ $\delta E_{\mathrm{ac}}, B_{\mathrm{ac}}$ : magnetic flux density or magnetic induction) can be obtained at zero bias field (self-biased CME effect). Systematic results on DME and CME hysteretic responses in three-phase laminate composites as a function of $H_{\text {bias }}$, are presented with analysis on resonant mode characteristics.

The configuration of three-phase ME composite and the DME and CME measurement setup has been described elsewhere. $^{9,13}$ First, $0.8 \quad\left[0.948 \quad\left(\mathrm{~K}_{0.5} \mathrm{Na}_{0.5} \mathrm{NbO}_{3}\right)-0.052\right.$ $\left.\left(\mathrm{LiSbO}_{3}\right)\right]-0.2\left[\mathrm{Ni}_{0.8} \mathrm{Zn}_{0.2} \mathrm{Fe}_{2} \mathrm{O}_{4}\right]$ (KNNLS-NZF) ME particulate composite disks with diameter of $10 \mathrm{~mm}$ and thickness of $0.5 \mathrm{~mm}$ were fabricated. The built-in magnetic bias was created in the sandwich structure (nickel $(\mathrm{Ni})$ plate with dimensions of $15 \times 15 \times 0.5 \mathrm{~mm}^{3}$ bonded between two KNNLS-NZF disks) due to difference in magnetic characteristics between Ni and NZF such as magnetization, susceptibility, coercivity, magnetostriction, and piezomagnetic coefficient. ${ }^{9,10}$ The electrical connections were made to operate the sample in bending mode configuration. In the DME measurement, $H_{a c}$ was provided by Helmholtz coil (DMEInput) to the laminate and then the induced voltage was measured from the sample (DME-Output). For CME measurement, $V_{a c}$ (CME-Input) was applied directly on the sample and then the induced magnetic flux density was measured by using Helmholtz coil (CME-Output). ${ }^{13}$

Figures 1(a) and 1(b) show the change in $\alpha_{\mathrm{DME}}$ and $\alpha_{\mathrm{CME}}$ coefficients in three-phase ME laminate as a function of $H_{\text {bias }}$ at off-resonance frequency of $1 \mathrm{kHz}$. The $\alpha_{\mathrm{DME}}$ shows a clear hysteretic behavior during $H_{\text {bias }}$ sweep with 


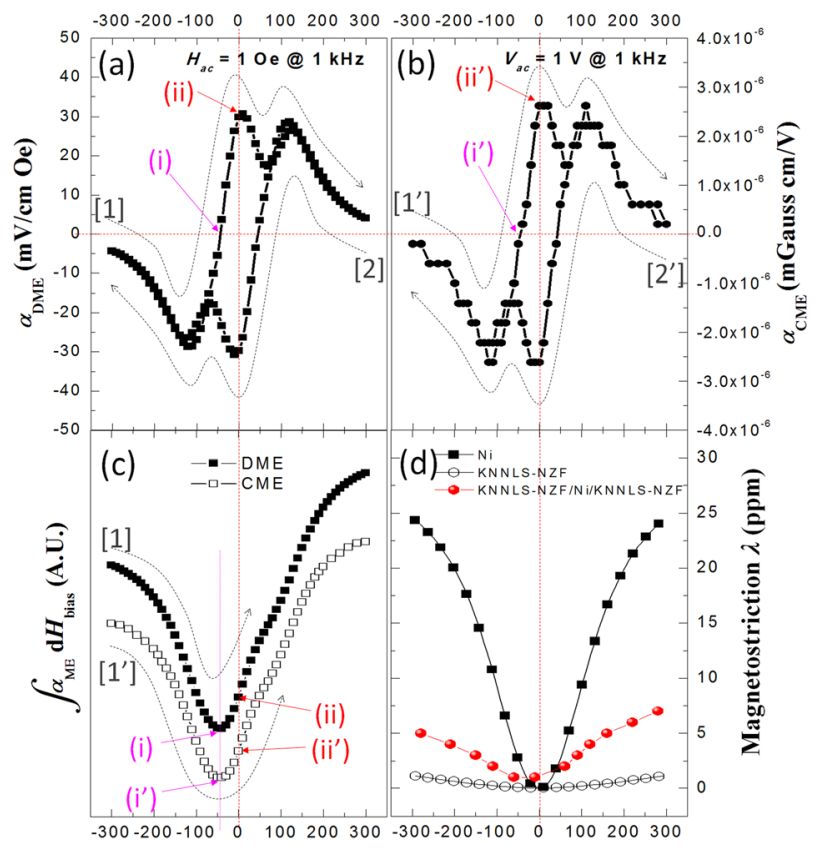

Magnetic bias field $\left(H_{\text {bias }}\right)$

FIG. 1. (Color online) (a) $\alpha_{\mathrm{DME}}$ and (b) $\alpha_{\mathrm{CME}}$ coefficients as a function of $H_{\text {bias }}$, (c) Integral values of $\alpha_{\mathrm{DME}}$ and $\alpha_{\mathrm{CME}}$ with respect to the $H_{\text {bias }}$, and (d) Magnetostriction of Ni, KNNLS-NZF, and bending mode KNNLS-NZF/Ni/ KNNLS-NZF as a function of $H_{\text {bias }}$.

remanent $\alpha_{\mathrm{DME}}$ value of $\sim \pm 30 \mathrm{mV} / \mathrm{cmOe}$ at $H_{\text {bias }}=0$ as shown in Fig. 1(a). In our previous study, we have shown that the shape of $\alpha_{\mathrm{DME}}$ can be controlled by adjusting the concentration of NZF (i.e., effect of built-in bias) or by modifying the structure of laminate. ${ }^{9,10}$ The laminate used in this work was optimized to show the peak $\alpha_{\mathrm{DME}}$ value at $H_{\text {bias }}=0$ for clear observation of self-biased effect. The trend of $\alpha_{\mathrm{DME}}$ with respect to the sweep directions (arrowed curves [1] and [2]) was the same. As shown in Fig. 1(b), the laminate showed CME effect with similar trend to that of DME effect. Remanent $\alpha_{\mathrm{CME}}$ of $\sim \pm 2.6 \times 10^{-6} \mathrm{mG} \cdot \mathrm{cm} / \mathrm{V}$ under $H_{\text {bias }}=0$ Oe was observed with hysteresis shape similar to $\alpha_{\mathrm{DME}}$ case clearly demonstrating that self-biased effect in this system is present not only for DME but also for CME effect. Theoretical investigations on DME and CME effects have revealed that ME coefficients are directly proportional to the piezomagnetic coefficient $q .{ }^{5,14}$ Moreover, similar tendency in hysteretic behavior between $\alpha_{\mathrm{DME}}$ and $\alpha_{\mathrm{CME}}$ reflects that the effect of $\lambda$ and/or $q$ of ferromagnetics on $H_{\text {bias }}$ dependence of ME effect is basically identical for both DME and CME cases. ${ }^{15}$ Considering the relation $\alpha$ $\propto q=d \lambda / d H_{\text {bias }}$, we estimated the effective $\lambda$ behavior by integrating $\alpha$ with respect to the $H_{\text {bias }}$ as shown in Fig. $1(\mathrm{c})$. Arrowed curves [1] and [1'] of Fig. 1(c) correspond to the same $H_{\text {bias }}$ sweep directions with [1] and [1'] in Figs. 1(a) and 1(b), respectively. Note that Fig. 1(c) shows only the qualitative nature of effective $\lambda$ behavior of the laminate and not quantitative values. Homogeneous ferromagnetic systems (individual NZF and Ni) usually show a symmetrical biquadratic $\lambda$ behavior with respect to $H_{\text {bias }}$ with $q=0$ at $H_{\text {bias }}=0$ as shown in Fig. 1(d). However, in our heterogeneous ferromagnetic system (NZF $+\mathrm{Ni}$ ), the position of $q=0$ was shifted from $H_{\text {bias }}=0$ to point (i) in Fig. 1(c) and the

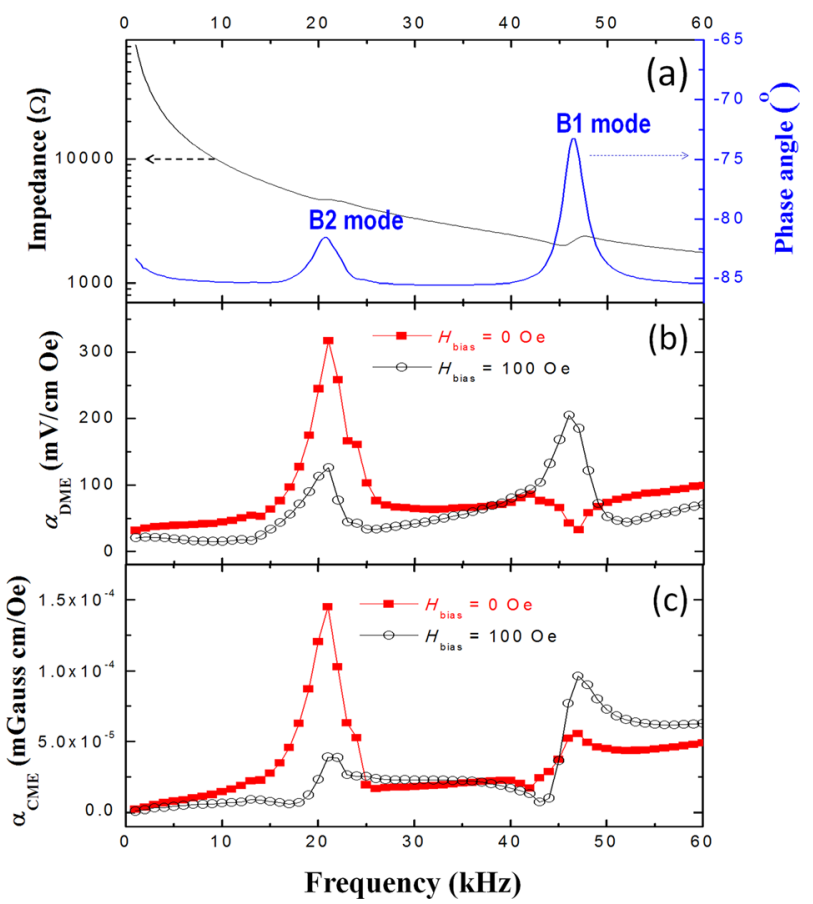

FIG. 2. (Color online) (a) Impedance and phase spectra for three-phase laminate under applied AC electric field, (b) $\alpha_{\mathrm{DME}}$ and (c) $\alpha_{\mathrm{CME}}$ as a function of frequency under applied $H_{\text {bias }}$ of 0 and $100 \mathrm{Oe}$.

tendency of $\lambda$ was not symmetric any more. In DME case, at the point (i) in Fig. 1(c), there is no change in $\lambda$ under small variation of magnetic field $\left(\delta H_{\mathrm{ac}}\right)$ implying that in this state magnetic domain rotation and domain wall migration become difficult. Therefore, no effective mechanical strain or stress can be delivered to ferroelectric phase resulting in zero-crossing $\alpha_{\text {DME }}$ point (i) in Fig. 1(a). Analogously, in CME case, since the generation of mechanical strain was restricted by domain pinning at point (i') in Fig. 1(c), quasipiezomagnetic effect was minimized showing zero-crossing $\alpha_{\mathrm{CME}}$ point (i') in Fig. 1(b). However, at zero-bias points (ii) and (ii') in Fig. 1(c), the slope of effective $\lambda$ has a finite value (the maximum value in our system). This large slope in $\lambda$ reflects easy domain rotation under $\delta H_{\mathrm{ac}}$ or $\delta T_{\mathrm{ac}},\left(T_{\mathrm{ac}}\right.$ : stress) resulting in strain generation and magnetization. Therefore, both DME and CME effect can be maximized under $H_{\text {bias }}=0$ at points (ii) and (ii') in Figs. 1(a) and 1(b) respectively. We believe that different magnetic properties of NZF and $\mathrm{Ni}$, including different $\lambda$ behavior in Fig. 1(d), and resultant interaction between built-in bias and $H_{\text {bias }}$ are at the origin of the shifted and non-symmetrical shape of effective $\lambda$ in Fig. 1(c) resulting in self-biased effect. Based on this hypothesis, we measured the $\lambda$ generated on the surface of KNN-NZF disk of the laminate during $H_{\text {bias }}$ sweep. As seen in Fig. 1(d), the measured $\lambda$ of the laminate clearly revealed the shift in curve with similar trend as obtained by integration value of $\alpha$ in Fig. 1(c). Although this measured $\lambda$ value may not reflect the exact magnitude of total $\lambda$ of the laminate, the shifted and unsymmetrical shape well explains the cause of self-biased ME phenomena.

It is well-known that ME response of composites is enhanced at electromechanical resonance of piezoelectric phase. Therefore, we investigated the frequency dependence of DME and CME response in three-phase laminate. Figure 2(a) 


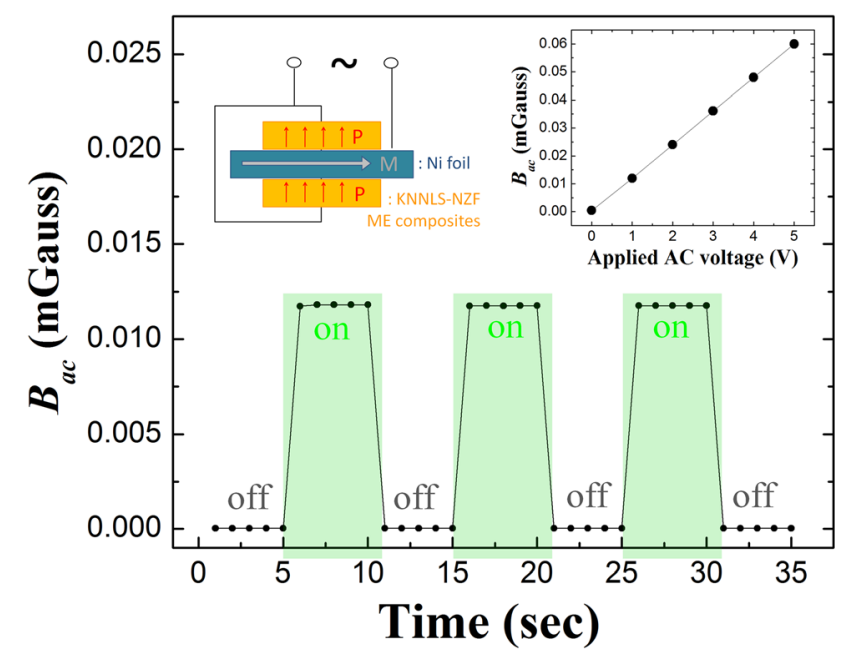

FIG. 3. (Color online) Magnetic flux density under $H_{\text {bias }}=0$ Oe measured by periodically applying $\mathrm{AC}$ voltage of $1 \mathrm{~V}$ at $21 \mathrm{kHz}$. Insets show the configuration of bending-mode three-phase laminate (KNNLS-NZF/Ni/ KNNLS-NZF) and the change in magnetic flux density with increasing AC voltage.

shows the impedance spectrum of the laminate. The first resonance peak for mechanical bending mode due to the electrical connection with $180^{\circ}$ current phase difference between top and bottom KNNs (B1 mode) was found at $47 \mathrm{kHz}$. However, another small resonance peak was observed at $21 \mathrm{kHz}$ which is not the B1 mode resonance. This additional bending vibration mode (B2 mode) is possibly due to the slight asymmetry of top and bottom KNN disks. Note that if the laminate had perfect structural and compositional symmetry, the voltage generation would be completely zero under longitudinal magnetic field due to the $180^{\circ}$ voltage phase difference between top and bottom KNNs. ${ }^{8}$ Figures 2(b) and 2(c) illustrate the DME and CME response of the laminate in the same frequency range under applied $H_{\text {bias }}=0$ and $100 \mathrm{Oe}$. Under zero $H_{\text {bias }}$ condition, both $\alpha_{\mathrm{DME}}$ and $\alpha_{\mathrm{CME}}$ values exhibited maximum enhancement at $21 \mathrm{kHz}$ and much weaker response at $47 \mathrm{kHz}$. However, under $H_{\text {bias }}=100 \mathrm{Oe}$, the maximum response was observed at $47 \mathrm{kHz}$ while smaller value was observed at $21 \mathrm{kHz}$ for both DME and CME cases. This opposite trend between $H_{\text {bias }}=0$ and 100 Oe cases indicates that (i) the bending motion from built-in bias (self-biased effect) corresponds to the B2 mode and does not include B1 mode vibration and (ii) under high $H_{\text {bias }}$, the built-in bias contribution for the laminate is reduced and overall vibration mode is dominated by the B1 bending mode. It should be noted that the laminate system used in this work does not have effective net magnetic gradient because of NZF/Ni/NZF symmetry (zero net built-in bias). Moreover, when no bending motion is incorporated into this structure, no self-biased effect is observed. ${ }^{9}$ These facts imply that initial bending strain is required to activate interaction between built-in bias and $\delta H_{\mathrm{ac}}$ or $\delta T_{\mathrm{ac}}$ in this system. The initial bending strain is created by B2 mode vibration which is from the structural and compositional asymmetry of KNNs. From this result, two important points can be noticed: (i) the frequency dependence of CME effect shows tendency similar to that of DME effect in both magnetic zero-biased and biased conditions and (ii) even though there is no initial magnetic gradient in the system, self-biased effect can be achieved by applying external bending strain.
The CME effect of the ME composites is of technological importance for the potential applications such as core-free magnetic flux control devices ${ }^{11}$ and electrically controlled magnetic memory devices. ${ }^{12}$ However, all the previous investigations on the $\mathrm{CME}$ effect have revealed that additional $H_{\text {bias }}$ is necessary for the maximum CME response alike the DME case. By eliminating additional $H_{\text {bias }}$, significant technical improvement of CME devices can be realized, e.g., the size reduction of devices and the exclusion of interference effect by electromagnet or permanent magnet. Figure 3 shows the magnetic flux density of the laminate generated by periodically applying $\mathrm{AC}$ voltage $\left(V_{\mathrm{ac}}\right)$ of $1 \mathrm{~V}$ at $21 \mathrm{kHz}$ under $H_{\text {bias }}=0$ Oe. The result clearly shows generation of magnetic flux density of $0.012 \mathrm{mG}$ with reliable repeatability in the absence of external $H_{\text {bias }}$. Furthermore, linear increase in magnetic flux was observed with increasing applied voltage as shown in the inset of Fig. 3 indicating promising application feasibility for tunable magnetic field generator.

In summary, the three-phase laminate composite consisting of heterogeneous ferromagnetics exhibited selfbiased DME and CME effects. The hysteretic behavior of ME response with remanent $\alpha$ values and zero-crossing characteristics of $\alpha$ were explained by the shift of magnetostriction curve of ferromagnetics caused by interaction between built-in bias and external magnetic bias. The result of this study predicts that self-biased ME effect may arise even in homogeneous ferromagnetic system when the external bending strain activates built-in bias. Further, magnitude of magnetic induction was controlled by applied voltage in the absence of external magnetic bias.

The authors gratefully acknowledge the financial support from National Science Foundation (Award No. 0757502) and DARPA Heterostructural Uncooled Magnetic Sensors (HUMS) program (Grant No. FA8650-09-1-7945).

${ }^{1}$ W. Eerenstein, N. D. Mathur, and J. F. Scott, Nature 442, 759 (2006).

${ }^{2}$ C. Israel, S. Kar-Narayan, and N. D. Mathur, Appl. Phys. Lett. 93, 173501 (2008).

${ }^{3}$ J. Ryu, A. V. Carazo, K. Uchino, and H. E. Kim, J. Electroceram. 7, 17 (2001).

${ }^{4}$ S. Priya, R. Islam, S. X. Dong, and D. Viehland, J. Electroceram. 19, 147 (2007).

${ }^{5}$ C. W. Nan, M. I. Bichurin, S. Dong, D. Viehland, and G. Srinivasan, J. Appl. Phys. 103, 031101 (2008).

${ }^{6}$ T. Wu, C. M. Chang, T. K. Chung, and G. Carman, IEEE Trans. Magn. 45, 4333 (2009).

${ }^{7}$ S. Dong, J. Zhai, J. Li, and D. Viehland, Appl. Phys. Lett. 89, 252904 (2006).

${ }^{8}$ S. K. Mandal, G. Sreenivasulu, V. M. Petrov, and G. Srinivasan, Appl. Phys. Lett. 96, 192502 (2010).

${ }^{9}$ S. C. Yang, C. S. Park, K.-H. Cho, and S. Priya, J. Appl. Phys. 108, 093706 (2010).

${ }^{10}$ S. C. Yang, C. W. Ahn, K.-H. Cho, and S. Priya, J. Am. Ceram. Soc. 94, 3889 (2011).

${ }^{11}$ Y. Jia, S. W. Or, H. L. W. Chan, X. Zhao, and H. Luo, Appl. Phys. Lett. 88, 242902 (2006).

${ }^{12}$ Y. Wang, F. Wang, S. W. Or, H. L. W. Chan, X. Zhao, and H. Luo, Appl. Phys. Lett. 93, 113503 (2008).

${ }^{13}$ S. Y. Chen, Q. Y. Ye, W. Miao, D. H. Wang, J. G. Wan, J. M. Liu, Y. W. Du, Z. G. Huang, and S. Q. Zhou, J. Appl. Phys. 107, 09D901 (2010).

${ }^{14}$ M. Lallart, Ferroelectrics-Physical Effect (InTech, Rijeka, Croatia, 2011), pp. 277-312.

${ }^{15}$ K.-H. Cho and S. Priya, Appl. Phys. Lett. 98, 232904 (2011). 